\title{
Exploring the brain through posterior hypothalamus surgery for aggressive behavior
}

\author{
Michele Rizzi, MD,1-3 Andrea Trezza, MD,,5 Giuseppe Messina, MD, ${ }^{1}$ \\ Alessandro De Benedictis, MD, PhD, ${ }^{4}$ Angelo Franzini, MD, ${ }^{1}$ and Carlo Efisio Marras, MD ${ }^{4}$ \\ ${ }^{1}$ Functional Neurosurgery Unit, Department of Neurosurgery, IRCCS Foundation "Istituto Neurologico Carlo Besta," Milan;

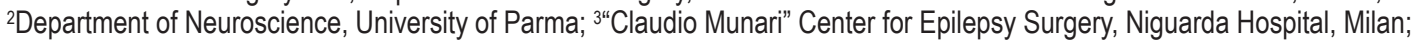 \\ ${ }^{4}$ Neurosurgery Unit, Department of Neuroscience and Neurorehabilitation, IRCCS Bambino Gesù Children's Hospital, Rome; \\ and ${ }^{5}$ Division of Neurosurgery, Department of Surgery and Translational Medicine, Milan Center for Neuroscience, University of \\ Milano-Bicocca, San Gerardo Hospital, Monza, Italy
}

\begin{abstract}
Neurological surgery offers an opportunity to study brain functions, through either resection or implanted neuromodulation devices. Pathological aggressive behavior in patients with intellectual disability is a frequent condition that is difficult to treat using either supportive care or pharmacological therapy. The bulk of the laboratory studies performed throughout the 19th century enabled the formulation of hypotheses on brain circuits involved in the generation of emotions. Aggressive behavior was also studied extensively. Lesional radiofrequency surgery of the posterior hypothalamus, which peaked in the 1970s, was shown to be an effective therapy in many reported series. As with other surgical procedures for the treatment of psychiatric disorders, however, this therapy was abandoned for many reasons, including the risk of its misuse. Deep brain stimulation (DBS) offers the possibility of treating neurological and psychoaffective disorders through relatively reversible and adaptable therapy. Deep brain stimulation of the posterior hypothalamus was proposed and performed successfully in 2005 as a treatment for aggressive behavior. Other groups reported positive outcomes using target and parameter settings similar to those of the original study. Both the lesional and DBS approaches enabled researchers to explore the role of the posterior hypothalamus (or posterior hypothalamic area) in the autonomic and emotional systems.
\end{abstract}

https://thejns.org/doi/abs/10.3171/2017.6.FOCUS17231

KEY WORDS posterior hypothalamus; aggressive behavior; deep brain stimulation; DBS; lesioning; autonomic system; emotional system

$\mathrm{N}$ EUROSURGERY offers a unique opportunity to invasively explore neurological functions. Resective neurosurgery generally enables the study of loss of functions, even though, in some cases, the decompression of a healthy brain (e.g., in the case of tumor and trauma surgery) or the removal of epileptogenic zones can induce a gain (or regain) of brain functions. ${ }^{13}$

\section{Neurosurgery as a Brain Exploration Tool}

"Lesional neurosurgery" and invasive brain stimula- tion enable us to cure symptoms by generating a therapeutic modification of already-altered encephalic circuits or regions. The acute and chronic use of these techniques elicits physiological and pathological responses that offer a therapeutic chance for patients..$^{24}$

These procedures also provide a unique opportunity to increase understanding of the functioning of the brain. This acquired knowledge can be integrated with that obtained from laboratory experiments and preoperative testing to better understand the pathogenic process of diseases and to formulate hypotheses on physiological processing. ${ }^{37}$

ABBREVIATIONS DBS = deep brain stimulation; ID = intellectual disability; NEFA = nonesterified fatty acid; OAS = Overt Aggression Scale; pHyp = posterior hypothalamus; VC/VS = ventral capsule and ventral striatum. 
For example, surgery of the posterior hypothalamic region has been reported to be an effective treatment in severe cases of aggressive behavior in patients with intellectual disability (ID), ${ }^{15,23,35,38,66}$ which enables us to formulate a hypothesis on disease pathogenesis and brain functioning. ${ }^{51}$

\section{The Disease}

The prevalence rate of challenging behavior in people with ID is reported to be as high as $45 \%$, but limited incidence data exist. ${ }^{22,32,40}$ Pathological aggressive behavior is one of the main reasons that people with ID are referred to specialist services. ${ }^{69}$ This fact is not surprising, because people with ID rarely refer themselves for psychological or psychiatric help. Rather, they are usually referred when their behavior becomes a problem for someone else. This also might be the reason that most of the research on psychotherapeutic interventions in people with ID deals with anger and aggression. ${ }^{69}$ Aggressive outbursts can prevent such patients from forming and maintaining relationships and can result in their exclusion from educational, work, and social settings and the breakdown of their living situation. $^{5}$

Pharmacological therapy can be effective for treating acute symptoms. ${ }^{3}$ However, it often fails to be effective in the long term. Such therapy includes neuroleptics, anticonvulsants, antidepressants, anxiolytics, and mood stabilizers. The paucity of effective pharmacological treatment for aggressive and disruptive behaviors has generated support for early-intervention strategies, mainly in early childhood. In this developmental period, the altered behavior is easier to treat by using appropriate educational and psychological methods. ${ }^{17}$

The most recent Diagnostic and Statistical Manual of Mental Disorders ${ }^{4}$ has a new chapter on disruptive, impulse-control, and conduct disorders, which includes all the emotional and behavioral self-control disorders.

\section{Historical Foreword: The Laboratory Studies}

In 1872, Charles Darwin published Expression of the Emotions in Man and Animals, in which he declared that animal emotions are homologous for human emotions. Many expressions (such as baring the teeth when angry) were considered rudimentary patterns of action, and a set of "basic" emotions was observed to be unvaried across species and cultures. ${ }^{16}$ According to Darwin's statement, animal emotions could be used to study human emotions.

During those years, eminent clinicians and scientists were engaged in a debate on the feasibility of localizing brain functions. In 1870, the Prussians Gustav Fritsch (1838-1927) and Eduard Hitzig (1838-1907) described the effects of direct current stimulation over different parts of the brain cortex of dogs. ${ }^{27}$ They stated that stimulation evoked contralateral motor responses and that stimulating different parts of the cortex activated different groups of muscles, from which a topographical map of movements of the body was laid out on the surface of the brain. At the National Hospital for the Paralyzed and Epileptic (Queen Square), in London, John Hughlings Jackson (1835-1911) hypothesized (after observing patients with motor sei- zures) that movements had a somatotopic organization and influenced David Ferrier (1843-1924) in his studies on stimulation mapping of the motor cortex in primates. ${ }^{63,71}$ At the same hospital in 1885, Victor Horsley (1857-1916) performed the first modern-day epilepsy surgery on a patient with focal motor (Jacksonian) seizures. He localized the motor cortex based on Jackson's somatotopic concept, aided, for the first time, by the use of intraoperative electrical stimulation. ${ }^{62}$

In contrast, in that same period, Friedrich Goltz (18341902), in Halle, Germany, and then in Strasbourg, France, held a unitary view of brain functions based on experiments on the dog's brain. Following his view, "all areas of the cortex [serve] all the same functions." This hypothesis fell into disuse after the famous International Medical Congress held in London in 1881. On that occasion, Ferrier elegantly demonstrated the localization of motor function in the primate cortex through ablation followed by postmortem dissection..$^{63}$ Goltz observed separately that complete extirpation of the neocortex in dogs provoked rage. ${ }^{31}$ This was the first documented observation on the neurobiology of raging behavior.

A few decades later, the American physiologist Walter Cannon (1871-1945) and his fellow Philip Bard (18981977) reproduced Goltz's experiments on dog decortication to demonstrate the purely central origin of emotions (Cannon-Bard theory). Bard and Rioch found that, in their opinion, rage-like behavior elicited by decortication disappeared after a cut through the rostral end of the superior colliculi and the posterior edge of the mammillary body in a dog's brain. Since that time, the caudal hypothalamus was supposed to be the "center" of rage responses once released from cortical control.,10,12 The observed rage was described as "sham," because it was not directed toward any specific eliciting stimulus. Bard and his colleagues first used surgical brain lesions to study emotions, and they proposed the posterior area of the hypothalamus to be a key region in rage generation. This rage response could be mediated through a cortical top-down control. Neocortical regions that control rage reactions were supposed to be multiple. The orbital cortex was observed to have a role in rage responses in both cats and monkeys. ${ }^{28,29}$ The efficacy of hypothalamic radiofrequency lesioning in aggressive rats was shown decades later. ${ }^{2}$

Another structure that was thought to play a role in rage generation and hypothalamic system modulation was the amygdala; in the case of amygdala surgery, contradictory results, with either rage or a taming attitude in both cats and monkeys, were observed..$^{11,58,64}$ In a famous article by James Papez (1883-1958) in 1937, the hypothalamus, anterior thalamic nuclei, hippocampus, and gyrus cinguli were supposed to play a central role in emotions. A few years later, Paul Yakovlev (1894-1983) proposed that the orbitofrontal cortex, insula, amygdala, and anterior temporal lobe form a network that underlies emotion and motivation..$^{70}$ The neuroscientist Paul MacLean (1913-2007) considered the Papez circuit and the network described by Yakovlev to be a whole cortical-subcortical structure, which he named the "visceral brain" and later renamed the "limbic system." "41

In 1932, on the basis of the work of Johann Paul Kar- 
plus (1866-1936) and Alois Kreill (1864-1928), the anatomist Beattie, from McGill University in Montreal, Canada, described for the first time the division of the cat hypothalamus into 3 cellular groups: 1) supra-optic, 2) tuber cinereum, and 3) supramammillary or posterior. The posterior hypothalamus (pHyp) would present with efferent fibers to the midbrain and reticular formation (hypothalamotegmental tract), which belongs to the autonomic system. Afferent fibers would come from the the mesolimbic dopaminergic pathway and from the medial and ventral thalamic nuclei. ${ }^{14}$ In that period, accumulated data indicated a role of the whole hypothalamus in autonomic responses; the posterior part was thought to be dedicated to the orthosympathetic response and the anterior part to parasympathetic activation. ${ }^{48,50}$ The Nobel laureate Walter Hess (1881-1973) stated that the central representation of the sympathetic activities was located in a region that involved the pHyp and rostral midbrain, designated the "dynamogene zone" or "ergotropic sektor." However, the anterior hypothalamus and surrounding preoptic and septal areas have a parasympathetic role (named "trophotrop endophylaktischer sektor"). The 2 areas were supposed to overlap each other. ${ }^{36}$ Indeed, Hess' experiments failed to confirm a clear-cut segregation of the different hypothalamic areas. In the same period, the anatomist Ban, in Osaka, Japan, studied the rabbit diencephalon and described the hypothalamus as being divided into 3 zones from the medial to lateral portions, following the Grünthal conception of "a, b, c Zellgebiete." 33 On the medial and lateral sides are parasympathetic zones (the a zone and the c zone), and Ban described a sympathetic area between them (the $b$ zone). In particular, he described the dorsal longitudinal fasciculus as the output of sympathetic activity. ${ }^{8}$ Based on Ban's studies, the concept of a rostrocaudal representation of autonomic responses switched to that of a mediolateral pattern.

Keiji Sano (1920-2011), a professor of neurosurgery at the University of Tokyo, had a broad knowledge of and admiration for all the aforementioned laboratory experiences (Fig. 1). ${ }^{52}$ As a surgeon, he was interested in understanding the translation of these observations into clinical practice. He also studied the psychic symptoms related to 297 limbic system tumors and found that lesions at the level of the posterior orbital area and the anterior hypothalamus generated euphoria, hyperactivity, or even irritability and rage, whereas lesions at the level of the cingulate gyrus, thalamus, and pHyp caused apathetic and somnolent symptoms. In contrast, temporal lobe symptoms could give rise to opposing clinical pictures..$^{52}$ Moreover, in those years at the University of Tokyo, the neurophysiologist Tokizane bestowed on the hypothalamus a central role in the activation of the limbic system. He and his group first described the cat pHyp as an archicortex (hippocampus)-activating region that also exerts influence on the neocortex via the reticular formation. ${ }^{65}$ Based on the concepts already discussed, Sano considered the pHyp, the anterior thalamic nuclei, the cingulate gyrus, and the hippocampus (the complete Papez circuit) to be the ergotropic circuit, whereas the trophotropic circuit consisted of the anterior hypothalamus, the posterior orbital area, the insula, the uncus, and the amygdala. According to his

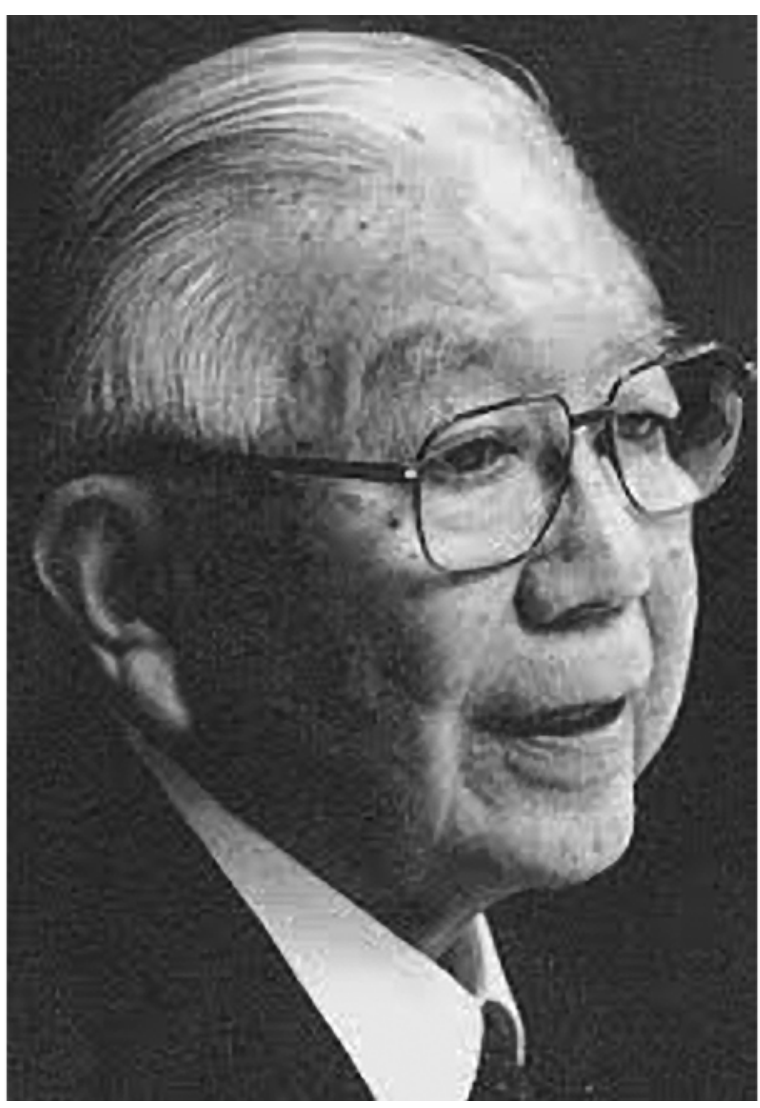

FIG. 1. Professor Keiji Sano, the pioneer of surgery for aggressive behavior. Photo courtesy of the Department of Neurosurgery, University of Tokyo.

description, these circuits had a central role in autonomic responses and also in experience and expression of emotions. In particular, he considered aggressive behavior the result of an unbalanced condition of the circuits, with the ergotropic circuit dominating. ${ }^{52}$

\section{Human Surgery: The Lesional Era and Neurophysiological Testing}

Sano was a pioneer in the treatment of aggressive behavior in the early days of stereotactic brain surgery. He proposed surgical lesioning of different targets at the ergotropic circuit level to calm the patient. Anterior cingulectomy, thalamotomy, fornicotomy, upper mesencephalic reticulotomy, and posteromedial hypothalamotomy were performed with this purpose (Table 1); posteromedial hypothalamotomy was the most effective procedure. Sano's explanation for the different outcomes was that surgery on lower-order areas produces more marked and lasting clinical effects than surgery on higher-order areas, because the overlapping of the ergotropic circuit with other circuits is more marked in the neocortex than the allocortex and in the allocortex than the diencephalon. ${ }^{52}$

Sano et al. ${ }^{52-55}$ reported the most detailed and consistent surgical series concerning patients with aggressive behavior treated by radiofrequency lesioning of the pHyp. Also worth mentioning is that other teams at that 
TABLE 1. The series of patients with aggressive behaviour treated by Sano

\begin{tabular}{lccccc}
\hline & Cases & Effective & Not Effective & Op. Deaths & Period of Observation \\
\hline Anterior cingulectomy & 14 & 7 & 4 & 3 & $6 \mathrm{y} 10 \mathrm{~m}-10 \mathrm{y} 4 \mathrm{~m}$ \\
\hline Thalamotomy & 4 & 2 & 1 & 1 & $2 \mathrm{y} 5 \mathrm{~m}-11 \mathrm{y}$ \\
\hline Fornicotomy & 21 & 15 & 4 & 2 & $2 \mathrm{y} 2 \mathrm{~m}-7 \mathrm{y} 2 \mathrm{~m}$ \\
\hline Upper mesencephalic reticulotomy & 6 & 4 & 2 & 0 & $2 \mathrm{y} 5 \mathrm{~m}-3 \mathrm{y} 2 \mathrm{~m}$ \\
\hline Posteromedial hypothalamotomy & 6 & 6 & 0 & 0 & $5 \mathrm{~m}-1 \mathrm{y} 4 \mathrm{~m}$ \\
\hline
\end{tabular}

Reproduced with permission from Sano: Neurol Med Chir (Tokyo) 4:112-142, 1962.

time proposed $\mathrm{pHyp}$ lesioning for the treatment of aggressive behavior. In Sapporo, Japan, Miyazaki et al. ${ }^{47}$ treated 4 patients (3 with ID) successfully with radiofrequency lesioning. In 1952 Spiegel and Wycis ${ }^{59}$ also reported on their experience of performing hypothalamotomy. They created surgical lesions in the lateral hypothalamic region near the subthalamic nucleus in schizophrenic patients. A group of authors from Chile also reported on their experiences. ${ }^{19}$ Sano and his team identified, through intraoperative pHyp-stimulation studies, the so-called b zone of Ban (its posterior part) between 1 and $5 \mathrm{~mm}$ from the wall of the third ventricle, which exhibited sympathetic responses. They developed a functional atlas of hypothalamic responses to stimulation using the Schaltenbrand-Bailey atlas. Following these considerations, Sano et al..$^{54}$ developed the concept of the "ergotropic triangle," in which sympathetic activity was elicited through intraoperative high-frequency stimulation, which became a sign of correct electrode placement. It is not clear if the stimulation covered the pHyp, the dorsal longitudinal fasciculus that arises there, or both.

Sano and his coworkers implanted electrodes at the pHyp level in 51 patients with aggressive behavior. They also implanted other electrodes at the level of the amygdala, hippocampus, temporal pole, and frontal lobe in the same surgical session. Scalp electroencephalography electrodes also were inserted. The procedure was performed on the contralateral side 7-10 days after the previous one. The following stimulation parameters were used: voltage 10-20 V; frequency $100 \mathrm{~Hz}$; pulse width $1000 \mu \mathrm{sec}$; and duration 5-10 seconds. A rise in blood pressure, tachycardia, and hyperpnea were observed after stimulation, principally $2-3 \mathrm{~mm}$ lateral from the wall of the third ventricle (Fig. 2). Pupillary dilation was seen in a slightly wider area than that after the previous stimulation. When stimulation was applied near the wall of the ventricle, an ipsilateral downward gaze in the patient was evident (stimulation ventrocaudal to this region resulted in an ipsilateral

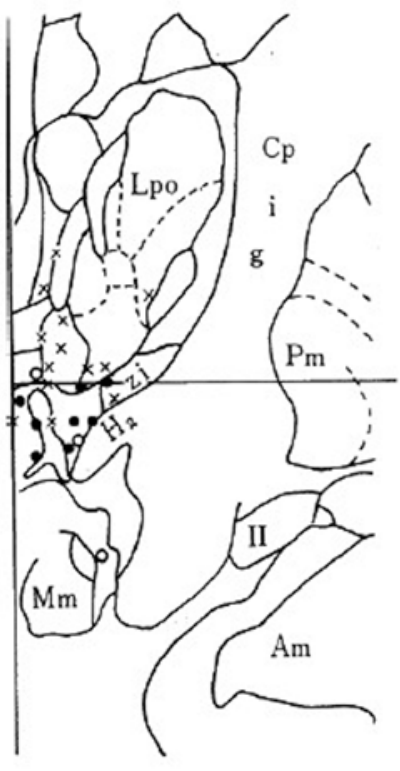

Fa 3.0

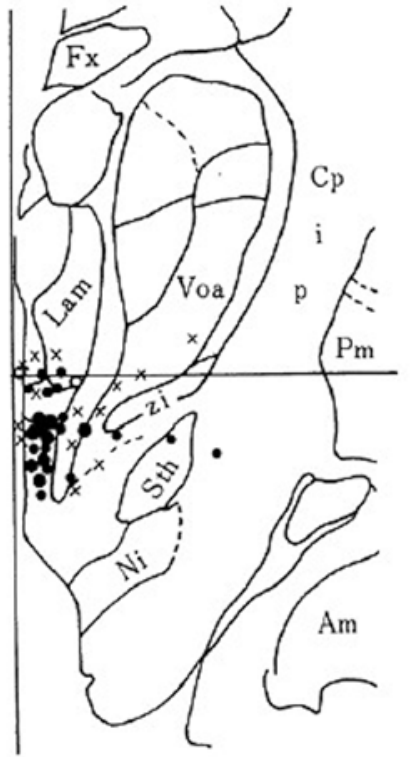

Fa 2.0

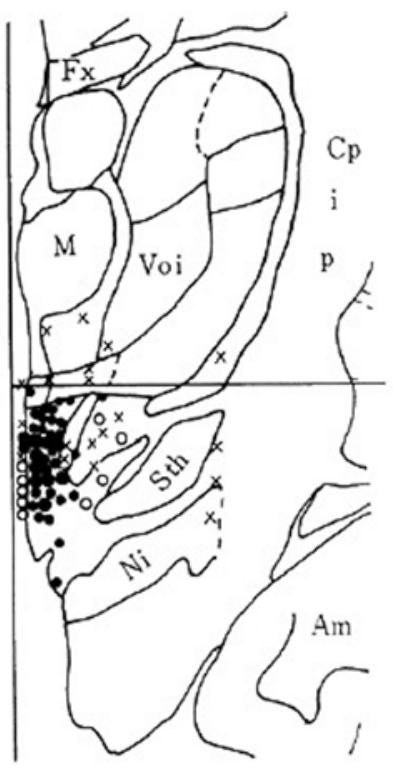

Fp 1.5

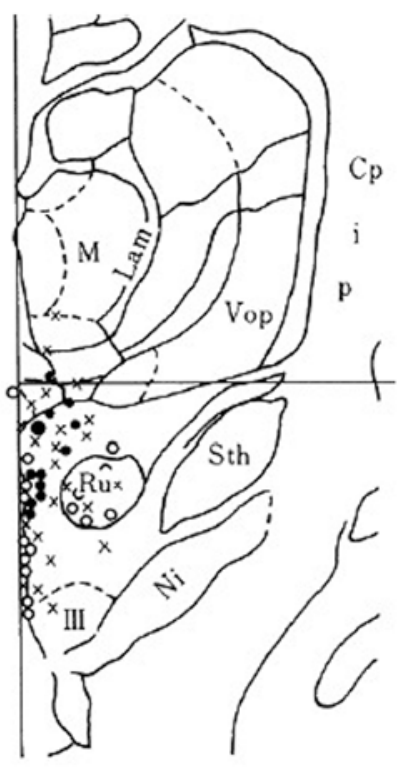

Fp 3.0

FIG. 2. Blood pressure (BP) changes by electrical stimulation. Black circles = rise in BP; open circles = decrease in BP; $X=n o$ change. $\mathrm{Am}=$ amygdaloid nuclei; $\mathrm{Cp}$ i $\mathrm{g}=$ capsula interna, genu; $\mathrm{Cp}$ i $\mathrm{p}=$ capsula interna, crus posterius; $\mathrm{Fx}=$ fornix; $\mathrm{H} 2=$ campus Forelii; II = tractus opticus; III = oculomotor nerve; Lam = lamella medialis thalami; $L p o=$ supranucleus lateropolaris thalami; $M=$ nucleus dorsomedialis; $\mathrm{Mm}$ = nucleus mammillaris medialis; $\mathrm{Ni}=$ substantia nigra; $\mathrm{Pm}=$ pallidium mediale; $\mathrm{Ru}=$ nucleus ruber; Sth = corpus subthalamicum; Voa = nucleus ventro-oralis anterior; Voi = nucleus ventro-oralis internus; Vop = nucleus ventrooralis posterior; Zi = zona incerta. Reprinted from Sano et al: J Neurosurg 33:689-707, 1970. Published with permission. 

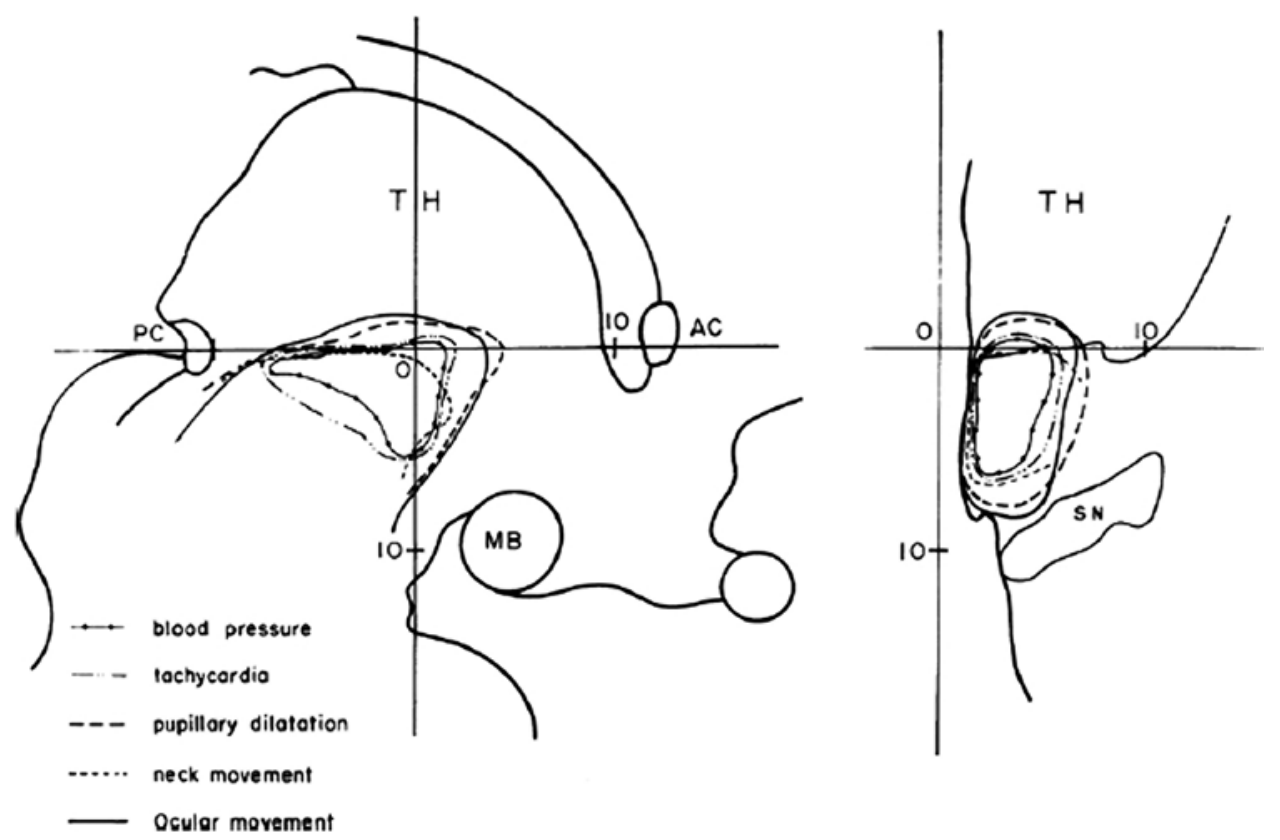

FIG. 3. Ergotropic (or Sano's) triangle. Summary of autonomic and somatomotor responses. $\mathrm{TH}=$ thalamus; $\mathrm{MB}=$ mammillary body; SN = subthalamic nucleus; IO = $10 \mathrm{~mm}$. Reprinted from Sano et al: J Neurosurg 33:689-707, 1970. Published with permission.

inward gaze, and that to the lateral portion, an opposite conjugate deviation). pHyp and medial subthalamic nucleus stimulation also evoked the contraction of mainly ipsilateral sternocleidomastoid and posterior neck muscles, probably because of stimulation of the medial longitudinal fasciculus. Red nucleus stimulation caused the lowering of blood pressure and pupillary constriction, probably because it is near the parasympathetic hypothalamic output. Because of these considerations, Sano defined the ergotropic triangle, known later as the Sano triangle, and described it, in the lateral view, as delimited by the midpoint of the anterior-posterior commissure line, rostral end of the aqueduct, and anterior border of the mammillary body (Fig. 3).

It is worth mentioning that, after stimulation, increased levels of nonesterified fatty acids (NEFAs) and growth hormone, electroencephalographic desynchronization at the level of the neocortex and the allocortex, and in some cases allocortex spiking, were observed.

Radiofrequency lesions were created at the area that showed a sympathetic response at a frequency of $1 \mathrm{MHz}$ and a power of $2-3 \mathrm{~W}$ for 3-4 minutes; the estimated size of the lesions was 3-4 mm. Sano et al. reported a followup duration of more than 1 year in 42 of the 51 patients. An improvement in aggressive behavior was found in $95 \%$ of the patients; this improvement was excellent in 12 patients and good in 28 patients. Sano et al. described markedly calm, passive, and tractable patients who were behaving with decreased spontaneity. The patients' IQ improved (even though values were not specified), probably because of their increased cooperation. Outcomes were not reported using validated scales (the article was published in 1970). The mecholyl test (responses to a parasympathomimetic drug) revealed a tendency toward parasympathicotonia. A general slight tendency to gain weight was seen also, together with a decrease in NEFA levels. However, in the subgroup of patients who gained weight, NEFA levels increased. Of these 42 patients, 22 also experienced seizures before surgery. After surgery, 1 patient became seizure free, and reductions in seizure intensity and frequency were experienced by another 9 patients $(41 \%)$ who were receiving the same or a smaller dosage of medications at more than 2 years of follow-up. ${ }^{54}$

In the 1970s and 1980s, other groups repeated Sano's procedures for the treatment of aggressive behavior $7,44,57,60$ or for indications such as "extreme" sexual behavior ${ }^{20}$ and alcohol and drug addiction ${ }^{20}$ and for the treatment of "erethic children." 5

\section{Human Surgery: The DBS Era}

Deep brain stimulation (DBS) of the pHyp has been proposed for the treatment of other disorders. Franzini and colleagues ${ }^{26}$ described, for the first time, remarkable results in the treatment of drug-resistant chronic cluster headache $(\mathrm{CCH})^{45}$ performed at the "Carlo Besta" Neurological Institute in Milan, Italy (Fig. 4). The choice of target was guided by PET scan activation of the pHyp during $\mathrm{CCH}$ attacks. ${ }^{39}$

Several reasons led researchers to consider the pHyp as a target for DBS to treat pathological aggressive behavior. First, the area that was targeted for DBS to treat $\mathrm{CCH}$ was the same as that identified by Sano et al..$^{54}$ for the treatment of aggressive behavior. Furthermore, the occasional onset of aggressive behavior during $\mathrm{CCH}$ attacks in some patients suggested a common involvement of the pHyp in the pathogenesis of both conditions. Moreover, experimental evidence for the existence of functional connections between the pHyp and other emotional structures (amygdala and the Papez circuit) has been reported. ${ }^{42}$ 


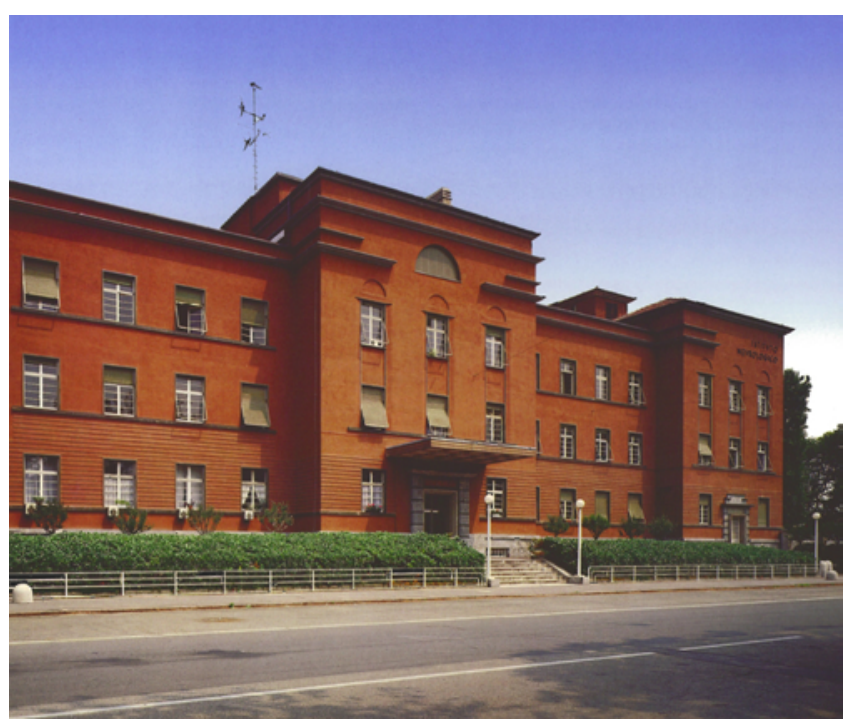

FIG. 4. "Carlo Besta" Neurological Institute in Milan, Italy, where DBS of the pHyp was performed for the first time. Reprinted with permission from the Press Office Fondazione I.R.C.C.S. Istituto Neurologico C. Besta.

Based on these data, Franzini et al. ${ }^{25}$ proposed bilateral DBS of the pHyp to treat aggressive behavior using a reversible procedure that was more conservative than lesional surgery (Fig. 5). They reported on 7 patients who had been affected by pathological aggressive and disruptive behavior, and the follow-up period ranged from 3 to 11 years; the patients benefited from high-frequency stimulation, and they experienced a marked reduction of aggressive outbursts until complete resolution..$^{23,25,26}$

Stimulation parameters in the series reported by Franzini et al. ${ }^{23}$ were as follows: frequency $185 \mathrm{~Hz}$; current amplitude 1-3 V; and pulse width 60-90 $\mu \mathrm{sec}$ with a monopolar configuration (case positive). The results were promising, including a mean improvement of $65 \%$ on the Overt Aggression Scale (OAS). ${ }^{72}$ In 1 case (postischemic aggressive behavior), no effect on aggressive behavior was noted. In contrast, an improvement in sleep pattern and a stable reduction in blood pressure values occurred. Another patient's violent behavior returned with the temporary shutdown of the battery. When stimulation was restored, however, the therapeutic effect was reduced despite parameter adjustments. These results were stable at followup. ${ }^{23,46} \mathrm{~A}$ recent review of outcomes revealed that the best results have been obtained in patients with a codiagnosis of ID (our unpublished data).

In recent years, other groups replicated the success of Franzini et al. Low-frequency and high-frequency stimulation with both monopolar and bipolar stimulation patterns were found equally beneficial in controlling dysfunctional behavior in patients. In 2008, Kuhn et al. ${ }^{38}$ treated a 22-year-old patient who developed self-aggressive behavior after a severe head injury, and after treatment, the patient's self-mutilation behavior disappeared. In the same period, another case of self-aggressive and heteroaggressive behavior was treated successfully with DBS of the pHyp. The peculiarity of the case was that a stable and significant clinical improvement was obtained

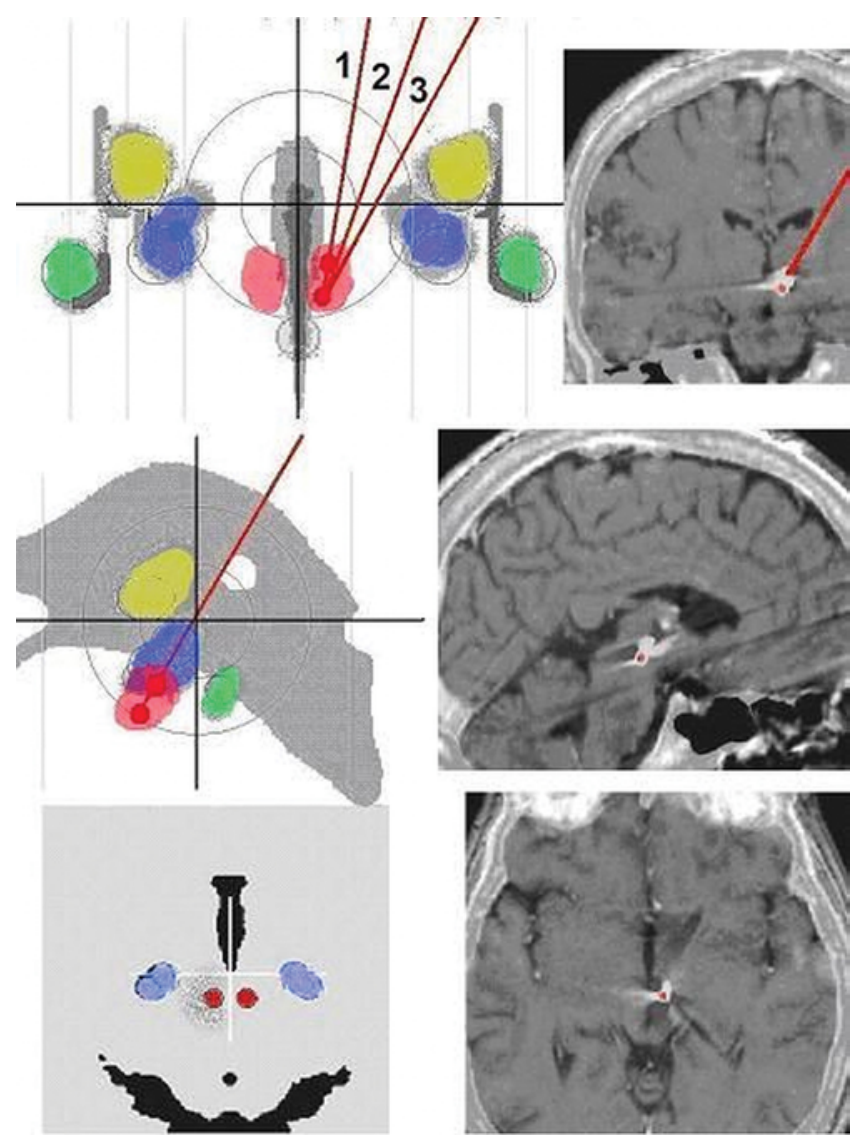

FIG. 5. Left: Virtual ventriculography plates showing the target (pHyp, pink and red) in the coronal (upper), sagittal (center), and axial (lower) sections as part of surgical planning. Trajectories are numbered 1, 2, and 3 in the anteroposterior ventricular representation and show the angles used to reach the target. Trajectories can change according to the ventricular shape and dimensions and the presence of vessels within the precoronal frontal cortex at the entry point. Right: Postoperative CT images merged with preoperative MR images showing the definitive location of an electrode in the coronal (upper), sagittal (center), and axial (lower) planes. Reprinted from Franzini et al: Neurosurg Focus 29(2):E13, 2010. Published with permission.

with different stimulation parameters-namely, a longer pulse width (450 $\mu \mathrm{sec})$ and a very low frequency $(15 \mathrm{~Hz})$. Moreover, the stimulation was monopolar on the left side and bipolar on the right side. ${ }^{35}$ In 2013 , Torres et al. ${ }^{66}$ presented a large series of patients with aggressive behavior and severe ID (1 patient had normal development until the age of 14 years). The Spanish group achieved similar clinical results when using low-frequency stimulation (15-60 $\mathrm{Hz})$ and a longer pulse width $(450 \mu \mathrm{sec})$ with low amplitude $(<1 \mathrm{~V})$ and when using high-frequency stimulation $(130-185 \mathrm{~Hz})$, a shorter pulse width $(60-210 \mu \mathrm{sec})$, and a higher current amplitude $(1.8-2.5 \mathrm{~V})$. These data were further confirmed by a group from Colombia who treated 5 patients with medically intractable aggressive behavior and seizures. The mean improvement on the OAS score was similar to that in the Franzini et al. study, and significant improvement in quality of life of the patients and caregivers was found. Seizure outcomes also were impressive. ${ }^{15}$

In all these series, adverse neurovegetative effects were 
TABLE 2. Experiences in DBS of the pHyp for the treatment of aggressive behavior

\begin{tabular}{|c|c|c|c|c|}
\hline Authors \& Year & $\begin{array}{l}\text { No. of } \\
\text { Patients }\end{array}$ & $\begin{array}{l}\text { FU } \\
\text { Duration }\end{array}$ & Stimulation Parameters at Last FU & Clinical Result \\
\hline Kuhn et al., 2008 & 1 & 4 mos & Monopolar: $130 \mathrm{~Hz}, 90 \mu \mathrm{sec}, 1.5 \mathrm{~V}$ & Complete resolution \\
\hline Hernando et al., 2008 & 1 & $18 \mathrm{mos}$ & Monopolar/bipolar: $15 \mathrm{~Hz}, 450 \mu \mathrm{sec}, 0.6-0.9 \mathrm{~V}$ & Persistent improvement \\
\hline Franzini et al., 2013 & 7 & $2-11$ yrs & Monopolar: $185 \mathrm{~Hz}, 60-90 \mu \mathrm{sec}, 1-3 \mathrm{~V}$ & $\begin{array}{l}\text { Mean } 65 \% \text { improvement on OAS (range } 0 \%- \\
100 \%) ; 50 \% \text { reduction in DRE (2 patients) }\end{array}$ \\
\hline Torres et al., 2013 & 6 & $6-82$ mos & $\begin{array}{l}\text { Monopolar/bipolar: } 15-185 \mathrm{~Hz}, 60-450 \mu \mathrm{sec} \text {, } \\
\text { 1.3-2.5 V }\end{array}$ & $\begin{array}{l}47 \% \text { improvement on ICAP scale for general ag- } \\
\text { gressiveness; } 30 \% \text { reduction in DRE ( } 1 \text { patient) }\end{array}$ \\
\hline $\begin{array}{l}\text { Benedetti-Isaac et al., } \\
\quad 2015\end{array}$ & 5 & $2-48 \mathrm{mos}$ & $\begin{array}{l}\text { Cyclic (1 min on, } 5 \text { min off): } 185 \mathrm{~Hz}, 90 \mu \mathrm{sec} \text {, } \\
2.4-3 \mathrm{~V}\end{array}$ & $\begin{array}{l}\text { Mean } 65 \% \text { improvement on OAS }(0 \%-100 \%) \\
\text { mean } 89.6 \% \text { seizure reduction }(50.8 \%-100 \%)\end{array}$ \\
\hline
\end{tabular}

DRE = drug-resistant epilepsy; FU = follow-up; ICAP = Inventory for Client and Agency Planning.

encountered when the stimulation amplitude was higher than $3 \mathrm{~V}$, which confirms what Sano found in the 1970s and might limit the therapeutic window of DBS. A summary of the aforementioned experiences is listed in Table 2.

Other experiences have highlighted the complexity of the neuronal networks involved in raging behavior, and some authors have proposed alternative targets for treating aggressiveness. Maley et al. ${ }^{43}$ in 2010 and Giordano et al. $^{30}$ in 2016 highlighted the role of the orbitofrontal cortex in the modulation of anger, and they performed DBS on the bilateral ventral capsule and ventral striatum ( $\mathrm{VC} /$ VS) region that resulted in significant clinical improvement. Thus, the relevance of the ergotropic circuit regained new relevance. Taira and colleagues ${ }^{61}$ reported a case of self-mutilating behavior successfully treated with DBS of the globus pallidus internus in a patient with Lesch-Nyhan syndrome and dystonia. The case suggested the possible involvement of motor circuits in the pathogenesis of selfaggressive syndromes. This result was confirmed and replicated by other authors in a few patients.,18

\section{Contribution of pHyp Surgery to the Neurological Sciences}

The authors of all the aforementioned laboratory studies on animals (mainly dogs, cats, and rats) considered the hypothalamus a key structure in the autonomic system. In stimulation studies, a rostrocaudal organization of the responses was hypothesized (sympathetic on the posterior side, parasympathetic on the anterior one). ${ }^{36} \mathrm{~A}$ second wave of studies developed by $\mathrm{Ban}^{8}$ considered mediolateral segregation on which sympathetic responses arose between the medial and lateral parasympathetic zones. Moreover, aggressive behavior produced by decortication, which caused a lack of cortical control, disappeared after a cut at the level of the "caudal" (posterior) hypothalamus in a dog's brain. ${ }^{10}$ The sympathetic system activation observed during episodes of raging behavior led to consideration of the hypothalamus as a structure that is deeply involved in both autonomic and emotional aspects.

These considerations led many groups, particularly the Sano group in Tokyo, to develop posterior radiofrequency hypothalamotomy for the treatment of aggressive behavior. Thanks to their stimulation and lesioning studies, Sano et al. helped clarify the multiple roles of the pHyp and provide clinical correlates.
The pHyp represents a low-order diencephalic region on which the limbic (emotional) and autonomic systems overlap..$^{54}$

\section{Autonomic System Insight}

Sano's acute stimulation of the pHyp (10-20 V, $100 \mathrm{~Hz}$, and $1000 \mu \mathrm{sec}$ for 5-10 seconds) at the level of the b zone of Ban produced mainly sympathetic responses, namely, a rise in blood pressure (Fig. 2), tachycardia, hyperpnea, and pupillary dilation. Hypothalamic radiofrequency lesioning was performed at this level, which tended to cause a decrease in sympathicotonia.

The anatomical correlate of these responses lies in the connection between the pHyp and the brainstem and spinal cord autonomic centers. In particular, the target of these neurophysiological and lesioning studies also could have been the fibers of the dorsal longitudinal fasciculus of Schutz, which arise from the pHyp.

Worth mentioning is that in the reported series, chronic stimulation (DBS) rarely induced transient sympathetic responses, probably because of the lower intensity of stimulation (1-3 V in our group) and the lower pulse widths $(60-177 \mu \mathrm{sec})$. The frequency of stimulation was high in both experiences $(100 \mathrm{~Hz}$ in the Sano et al. study and 130$185 \mathrm{~Hz}$ in DBS studies). ${ }^{22,52}$

\section{Emotional System Insight}

Sano described hypothalamotomy as "sedative."

The patient became markedly calm, passive, and tractable, showing decreased spontaneity. These changes were long-lasting, although spontaneity returned to a considerable extent in 1 month. Sometimes somnolence was noted in the first 7 to 10 days after the operation. Intelligence was not impaired by this operation, at least at the ordinary intelligence test administered more than 2 months after the operation. On the contrary, amelioration of the intelligence quotient was noted in about half of the cases; this may be because the patients became cooperative after hypothalamotomy. ${ }^{52}$

The described experience is similar to that experienced by our group and described in other reports. ${ }^{23}$ In our experience, the patients who underwent DBS became calmer, antipsychotic drugs were reduced, and, in some cases, the patients had an opportunity to be reintegrated partially or totally with their family as a result of their markedly reduced aggressive behavior. In our series, before surgery, 
all the patients presented with severe ID or a severe acquired cognitive problem. For this reason, cognitive testing was not possible.

pHyp lesioning and high-frequency DBS result in decreased activation of the emotional system, which exerts a strong effect, especially on the experience of rage. MacLean ${ }^{42}$ considered the pHyp part of the limbic system, whereas the Tokizane et al. ${ }^{65}$ studies on cat brains revealed that the pHyp activates the archicortex (hippocampus). Thus, the pHyp was considered to have a central role in activating the limbic system.

\section{From the Target to the Circuit: Extending Our Knowledge}

Given that the pHyp belongs to at least 2 different systems (autonomic and emotional), other brain structures might be involved and considered suitable targets for the treatment of aggressive behavior. DBS of the VC/VS has been performed successfully in 3 cases of aggressive behavior. ${ }^{30,34,43}$ This region is supposed to belong to the "emotional brain," a circuit that includes the anterior cingulate cortex, orbitofrontal cortex, amygdala, and insular cortex (similar to the visceral brain or limbic system described by MacLean ${ }^{42}$ ). Given the supposed beneficial result of DBS to this area and the hypothalamic area in the treatment of aggressive behavior, the connections between these 2 structures have been studied. ${ }^{51}$

The only described direct anatomophysiological link between these 2 regions is the mesolimbic dopaminergic pathway, the so-called reward pathway, which has been studied extensively in relation to addiction disorders and the positive symptoms of schizophrenia. A role of the mesolimbic pathway in aggression was proposed by the psychologists Panksepp and Zellner ${ }^{49}$ based on animal studies.

Both the VC/VS and the pHyp represent low-order structures under fine neocortical control. ${ }^{56}$

\section{Conclusions}

Challenging behavior, especially aggression, is present in half of the people with ID. The pharmacological treatment and dedicated community structures sometimes fail to control aggression, which results in negative consequences. ${ }^{5,22}$

In the past, posterior hypothalamotomy was performed successfully for the treatment of severe aggressive behavior, but almost all the "psychosurgical" procedures fell into disuse at the beginning of the 1970s because they were perceived to be a brain-disabling therapy or used to control minority groups or because of the worldwide diffusion of antipsychotic drugs as an effective nonsurgical therapy. ${ }^{67,68}$

DBS has represented a new treatment modality for severe neurological (e.g., Parkinson disease, dystonia, tremor) and psychiatric (e.g., obsessive-compulsive disorders) disorders in the last decades. DBS prevents a definitive lesion of the brain and is relatively reversible and adaptable to clinical conditions. For these reasons, it has been used successfully as a palliative therapy in some rare cases in which pharmacological therapy and social measures were not enough to control aggressive behavior. ${ }^{15,23}$

Both the lesional and DBS experiences have enabled us to understand the role of specific brain structures and to formulate reliable hypotheses on the physiology of the brain and the pathophysiology of aggressive behavior. These data are of paramount relevance, together with those coming from clinical and animal laboratory studies.

The pHyp can be considered a key structure for both the autonomic nervous and emotional (limbic) systems. Acute and chronic stimulation of the pHyp, with different parameters and radiofrequency lesioning, enable us to elicit or suppress autonomic features and to deeply influence the emotional status of a patient. The observed endocrinological and epileptological modifications give $\mathrm{pHyp}$ a role in those fields too.

\section{Acknowledgments}

We thank Prof. Orsola Gambini (San Paolo Hospital, Milan, and University of Milan), Prof. Stefano Vicari (Bambino Gesù Children's Hospital, Rome), Stefano Cavallari, MD (S. Spirito Hospital, Rome), and Laura Giustolisi, MD (Disability Unit, ASL Roma 1), for taking part in patient management. We also thank Paola Volpi, MS, for language revision.

\section{References}

1. Abel TJ, Dalm BD, Grossbach AJ, Jackson AW, Thomsen T, Greenlee JDW: Lateralized effect of pallidal stimulation on self-mutilation in Lesch-Nyhan disease. J Neurosurg Pediatr 14:594-597, 2014

2. Adams DB: Defence and territorial behaviour dissociated by hypothalamic lesions in the rat. Nature 232:573-574, 1971

3. Aman MG, De Smedt G, Derivan A, Lyons B, Findling RL: Double-blind, placebo-controlled study of risperidone for the treatment of disruptive behaviors in children with subaverage intelligence. Am J Psychiatry 159:1337-1346, 2002

4. American Psychological Association: Diagnostic and Statistical Manual of Mental Disorders, ed 5. Washington, DC: American Psychological Association, 2013

5. Antonacci DJ, Manuel C, Davis E: Diagnosis and treatment of aggression in individuals with developmental disabilities. Psychiatr Q 79:225-247, 2008

6. Arjona VE: Sterotactic hypothalamotomy in erethic children. Acta Neurochir (Wien) Suppl 21:185-191, 1974

7. Balasubramaniam V, Kanaka TS: Amygdalotomy and hypothalamotomy-a comparative study. Confin Neurol 37:195-201, 1975

8. Ban T: The septo-preoptico-hypothalamic system and its autonomic function. Prog Brain Res 21:1-43, 1966

9. Bard P: Central nervous mechanisms for emotional behavior patterns in animals. Res Publ Assoc Res Nerv Ment Dis 19:190-218, 1939

10. Bard P: A diencephalic mechanism for the expression of rage with special reference to the sympathetic nervous system. Am J Physiol 84:490-515, 1928

11. Bard P, Mountcastle VB: Some forebrain mechanisms involved in expression of rage with special reference to suppression of angry behavior. Res Publ Assoc Res Nerv Ment Dis 27:362-404, 1948

12. Bard P, Rioch DM: A study of four cats deprived of neocortex and additional portions of the forebrain. Bull Johns Hopkins Hosp 60:73-147, 1937

13. Baxendale S, Thompson PJ, Duncan JS: Improvements in memory function following anterior temporal lobe resection for epilepsy. Neurology 71:1319-1325, 2008

14. Beattie J, Brow GR, Long CNH: Physiological and anatomical evidence for the existence of nerve tracts connecting the hypothalamus with spinal sympathetic centres. Proc R Soc Lond B Biol Sci 106:153-275, 1930

15. Benedetti-Isaac JC, Torres-Zambrano M, Vargas-Toscano A, 
Perea-Castro E, Alcalá-Cerra G, Furlanetti LL, et al: Seizure frequency reduction after posteromedial hypothalamus deep brain stimulation in drug-resistant epilepsy associated with intractable aggressive behavior. Epilepsia 56:1152-1161, 2015

16. Dalgleish T: The emotional brain. Nat Rev Neurosci 5:583-589, 2004

17. Davies L, Oliver C: The age related prevalence of aggression and self-injury in persons with an intellectual disability: a review. Res Dev Disabil 34:764-775, 2013

18. Deon LL, Kalichman MA, Booth CL, Slavin KV, GaeblerSpira DJ: Pallidal deep-brain stimulation associated with complete remission of self-injurious behaviors in a patient with Lesch-Nyhan syndrome: a case report. J Child Neurol 27:117-120, 2012

19. Diaz Perez G, Chiorino R, Donoso P, Aranda L, Asenjo A: [Posterior hypothalamotomy using the stereotaxic method in the treatment of erethism and aggressiveness.] Neurocirugia 26:12-18, 1968 (Span)

20. Dieckmann G, Schneider H: Influence of stereotactic hypothalamotomy on alcohol and drug addiction. Appl Neurophysiol 41:93-98, 1978

21. Dieckmann G, Schneider-Jonietz B, Schneider H: Psychiatric and neuropsychological findings after stereotactic hypothalamotomy, in cases of extreme sexual aggressivity. Acta Neurochir Suppl (Wien) 44:163-166, 1988

22. Emerson E, Kiernan C, Alborz A, Reeves D, Mason H, Swarbrick R, et al: The prevalence of challenging behaviors: a total population study. Res Dev Disabil 22:77-93, 2001

23. Franzini A, Broggi G, Cordella R, Dones I, Messina G: Deep-brain stimulation for aggressive and disruptive behavior. World Neurosurg 80:29.e11-29.e14, 2013

24. Franzini A, Cordella R, Messina G, Marras CE, Romito LM, Albanese A, et al: Targeting the brain: considerations in 332 consecutive patients treated by deep brain stimulation (DBS) for severe neurological diseases. Neurol Sci 33:1285-1303, 2012

25. Franzini A, Marras C, Ferroli P, Bugiani O, Broggi G: Stimulation of the posterior hypothalamus for medically intractable impulsive and violent behavior. Stereotact Funct Neurosurg 83:63-66, 2005

26. Franzini A, Messina G, Cordella R, Marras C, Broggi G: Deep brain stimulation of the posteromedial hypothalamus: indications, long-term results, and neurophysiological considerations. Neurosurg Focus 29(2):E13, 2010

27. Fritsch G, Hitzig E: Über die elektrische Erregbarkeit des Grosshirns. Arch Anat Physiol Wissen 37:300-332, 1870

28. Fulton JF, Ingraham FD: Emotional disturbances following experimental lesions of the base of the brain (pre-chiasmal). J Physiol 67:27-28, 1929

29. Fulton JF: Frontal Lobotomy and Affective Behavior. A Neurophysiological Analysis. New York: Norton, 1951

30. Giordano F, Cavallo M, Spacca B, Pallanti S, Tomaiuolo F, Pieraccini F, et al: Deep brain stimulation of the anterior limb of the internal capsule may be efficacious for explosive aggressive behaviour. Stereotact Funct Neurosurg 94:371-378, 2016

31. Goltz F: Ueber die Verrichtunger des Grosshirns. Archiv für Physiologie 42:1-49, 1881

32. Grey I, Pollard J, McClean B, MacAuley N, Hastings R: Prevalence of psychiatric diagnoses and challenging behaviors in a community-based population of adults with intellectual disability. J Ment Health Res 3:210-222, 2010

33. Grünthal E: Der Zellaufbau des Hypothalamus beim Hunde. Ztschr Neurol Psychiat 120:157-177, 1929

34. Harat M, Rudaś M, Zieliński P, Birska J, Sokal P: Deep brain stimulation in pathological aggression. Stereotact Funct Neurosurg 93:310-315, 2015

35. Hernando V, Pastor J, Pedrosa M, Peña E, Sola RG: Low- frequency bilateral hypothalamic stimulation for treatment of drug-resistant aggressiveness in a young man with mental retardation. Stereotact Funct Neurosurg 86:219-223, 2008

36. Hess CW: Walter R. Hess (17.3.1881-12.8.1973). Schweiz Arch Neurol Psychiatr 4:255-261, 2008

37. Kringelbach ML, Jenkinson N, Owen SLF, Aziz TZ: Translational principles of deep brain stimulation. Nat Rev Neurosci 8: $623-635,2007$

38. Kuhn J, Lenartz D, Mai JK, Huff W, Klosterkoetter J, Sturm $\mathrm{V}$ : Disappearance of self-aggressive behavior in a braininjured patient after deep brain stimulation of the hypothalamus: technical case report. Neurosurgery 62:E1182, 2008

39. Leone M, Franzini A, Bussone G: Stereotactic stimulation of posterior hypothalamic gray matter in a patient with intractable cluster headache. N Engl J Med 345:1428-1429, 2001

40. Lowe K, Allen D, Jones E, Brophy S, Moore K, James W: Challenging behaviours: prevalence and topographies. J Intellect Disabil Res 51:625-636, 2007

41. MacLean PD: The limbic system and its hippocampal formation; studies in animals and their possible application to man. J Neurosurg 11:29-44, 1954

42. MacLean PD: Psychosomatic disease and the visceral brain; recent developments bearing on the Papez theory of emotion. Psychosom Med 11:338-353, 1949

43. Maley JH, Alvernia JE, Valle EP, Richardson D: Deep brain stimulation of the orbitofrontal projections for the treatment of intermittent explosive disorder. Neurosurg Focus 29(2):E11, 2010

44. Matera R, Ríos E, Barbosa E, Bianucci MA, Yaria J: [Stereotaxic hypothalamotomy in aggressiveness.] Acta Neurol Latinoam 18:96-99, 1972 (Span)

45. May A, Bahra A, Büchel C, Frackowiak RSJ, Goadsby PJ: Hypothalamic activation in cluster headache attacks. Lancet 352:275-278, 1998

46. Messina G, Islam L, Cordella R, Gambini O, Franzini A: Deep brain stimulation for aggressive behavior and obsessive-compulsive disorder. J Neurosurg Sci 60:211-217, 2016

47. Miyazaki Y, Hirai H, Nakamura J, Matsumoto S, Sato A: Posterior hypothalamotomy for aggressive behavior. Neurol Med Chir (Tokyo) 7:281, 1965

48. Murphy JP, Gellhorn E: Further investigations on diencephalic-cortical relations and their significance for the problem on emotion. J Neurophysiol 8:431-447, 1945

49. Panksepp J, Zellner MR: Towards a neurobiologically based unified theory of aggression. Rev Int Psychol Soc 17:37-61, 2004

50. Ranson SW: The hypothalamus; its significance for visceral innervation and emotional expression. Trans Stud Coll Phys Phila 2:222-242, 1934

51. Rizzi M, Marras CE: Deep brain stimulation for the treatment of aggressive behaviour: considerations on pathophysiology and target choice. Stereotact Funct Neurosurg 95:114-116, 2017

52. Sano K: Sedative neurosurgery with special reference to posteromedial hypothalamotomy. Neurol Med Chir (Tokyo) 4:112-142, 1962

53. Sano K, Mayanagi Y: Posteromedial hypothalamotomy in the treatment of violent, aggressive behaviour. Acta Neurochir Suppl (Wien) 44:145-151, 1988

54. Sano K, Mayanagi Y, Sekino H, Ogashiwa M, Ishijima B: Results of stimulation and destruction of the posterior hypothalamus in man. J Neurosurg 33:689-707, 1970

55. Sano K, Yoshioka M, Ogashiwa M, Ishijima B, Ohye C: Postero-medial hypothalamotomy in the treatment of aggressive behaviors. Confin Neurol 27:164-167, 1966

56. Schoene-Bake JC, Parpaley Y, Weber B, Panksepp J, Hurwitz TA, Coenen VA: Tractographic analysis of historical lesion surgery for depression. Neuropsychopharmacology $35: 2553-2563,2010$ 
57. Schvarcz JR, Driollet R, Rios E, Betti O: Stereotactic hypothalamotomy for behaviour disorders. J Neurol Neurosurg Psychiatry 35:356-359, 1972

58. Spiegel EA, Miller HR, Oppenheimer MJ: Forebrain and rage reactions. J Neurophysiol 3:538-548, 1940

59. Spiegel EA, Wycis HT: Principles and application of stereoencephalotomy. Brain Nerve 4:129-137, 1952

60. Sramka M, Nádvorník P: Surgical complication of posterior hypothalamotomy. Confin Neurol 37:193-194, 1975

61. Taira T, Kobayashi T, Hori T: Disappearance of self-mutilating behavior in a patient with Lesch-Nyhan syndrome after bilateral chronic stimulation of the globus pallidus internus. Case report. J Neurosurg 98:414-416, 2003

62. Tan TC, Black PM: Sir Victor Horsley (1857-1916): pioneer of neurological surgery. Neurosurgery 50:607-612, 2002

63. Taylor CSR, Gross CG: Twitches versus movements: a story of motor cortex. Neuroscientist 9:332-342, 2003

64. Thomson AF, Walker AE: Behavioral alterations following lesions of the medial surface of the temporal lobe. Fol Psychiat Neurol Neurochir Neerl 53:444-452, 1950

65. Tokizane T, Kawamura H, Imamura G: [Effect oh hypothalamic stimulation on the activity of the paleocortex and archicortex.] Prensa Med Argent 51:283-285, 1964 (Span)

66. Torres CV, Sola RG, Pastor J, Pedrosa M, Navas M, GarcíaNavarrete E, et al: Long-term results of posteromedial hypothalamic deep brain stimulation for patients with resistant aggressiveness. J Neurosurg 119:277-287, 2013

67. United States Department of Health, Education, and Welfare: Determination of the Secretary regarding the recommendations on psychosurgery of the National Commission for the Protection of Human Subjects of Biomedical and Behavioral Research. Fed Regist 43(221):53241-53244, 1978

68. Valenstein E: The Practice of Psychosurgery: A Survey of the Literature (1971-1976). Washington, DC: United States Government Printing Office, 1977
69. Willner P: The neurobiology of aggression: implications for the pharmacotherapy of aggressive challenging behaviour by people with intellectual disabilities. J Intellect Disabil Res 59:82-92, 2015

70. Yakovlev PI: Motility, behavior and the brain; stereodynamic organization and neural coordinates of behavior. J Nerv Ment Dis 107:313-335, 1948

71. York GK III, Steinberg DA: Hughlings Jackson's neurological ideas. Brain 134:3106-3113, 2011

72. Yudofsky SC, Silver JM, Jackson W, Endicott J, Williams D: The Overt Aggression Scale for the objective rating of verbal and physical aggression. Am J Psychiatry 143:35-39, 1986

\section{Disclosures}

Dr. Rizzi reports personal fees from W.I.S.E. Biotech and nonfinancial support from Medtronic unrelated to the submitted work. The other authors report no conflict of interest concerning the materials or methods used in this study or the findings specified in this paper.

\section{Author Contributions}

Conception and design: Rizzi, Messina. Acquisition of data: Rizzi, Trezza. Analysis and interpretation of data: Rizzi, Trezza, De Benedictis. Drafting the article: Rizzi, Trezza. Critically revising the article: all authors. Reviewed submitted version of manuscript: Rizzi, Trezza, Messina. Approved the final version of the manuscript on behalf of all authors: Rizzi. Study supervision: Franzini, Marras.

\section{Correspondence}

Michele Rizzi, "Claudio Munari” Center for Epilepsy Surgery, Niguarda Hospital, Piazza Ospedale Maggiore 3, Milan 20162, Italy. email: michele.rizzi@live.it. 